\title{
Suitability of the Composite Made of Multi Wall Carbon Nanotubes-Polyvinylpyrrolidone for Culturing Invertebrate Helix aspersa Neurons
}

\author{
Juan Bernal-Martinez ${ }^{1 *}$, Rafael Godínez-Fernández¹, Alfredo Aguilar-Elguezabal² \\ ${ }^{1}$ Biophysical Laboratory, Electrical Engineering Department, Universidad Autónoma Metropolitana Iztapalapa, México City, \\ México \\ ${ }^{2}$ Department of Engineering and Chemistry of Materials, Centro de Investigación y Materiales Avanzados S.C., Chihuahua, \\ México \\ Email: *drjuanbernal@hotmail.com
}

How to cite this paper: Bernal-Martinez, J., Godínez-Fernández, R. and Aguilar-Elguezabal, A. (2017) Suitability of the Composite Made of Multi Wall Carbon Nanotubes-Polyvinylpyrrolidone for Culturing Invertebrate Helix aspersa Neurons. Journal of Materials Science and Chemical Engineering, 5, 41-50.

https://doi.org/10.4236/msce.2017.52005

Received: January 7, 2017

Accepted: February 14, 2017

Published: February 17, 2017

Copyright $\odot 2017$ by authors and Scientific Research Publishing Inc. This work is licensed under the Creative Commons Attribution International License (CC BY 4.0).

http://creativecommons.org/licenses/by/4.0/

\begin{abstract}
Carbon nanotubes have been used as scaffolds for tissue engineering. However, the identification of these nanomaterials disperses in biological solutions and their direct interaction with nerve cells is still controversial. The aim of this work is to characterize the topographic and ultra-structural distribution of the composite made of multi wall carbon nanotubes-polyvinylpyrrolidone (MWCNTs-PVP) deposited on the Helix aspersa neurons and over glass coverslip. Scanning Electron Microscopy (SEM) and Confocal Microscopy (CM) studies were done to analyze the properties of such MWCNTs-PVP composite. The cerebral ganglion of Helix aspersa was treated and incubated with MWCNTs-PVP, fixing it in paraformaldehyde at $4 \%$ and was observed with SEM and CM. Although the nanotubes were not labeled or stained with fluorescent compounds, the MWCNTs-PVP deposited on glass and on nerve cells, was observed by the confocal microscope in the reflection mode. In SEM studies, it was observed that MWCNTs-PVP was attached to the surface on neurons. Moreover, in CM studies, it was possible to observe that MWCNTs-PVP was attached to the neuronal membrane, crossing the cell membrane and getting into the cytoplasm. These results support the hypothesis that carbon nanotubes interact with the neuronal cell membrane and can be useful for neuronal tissue engineering. In addition, these results open new alternatives for toxicological studies, in order to elucidate the cytotoxicity of MWCNTs-PVP composite in neurons and other excitable cells.
\end{abstract}

\section{Keywords}

Multi Wall Carbon Nanotubes, Functional Scaffolds, Neurons, Helix aspersa, Tissue Engineering, Brain Repair 


\section{Introduction}

It has been proposed that carbon nanotubes are good scaffolds for cell culture, tissue engineering and tissue repair [1] [2] [3]. However, there are controversial reports about the toxicity of carbon nanotubes and the suitability of such nanomaterials as functional scaffolds [4]-[10]. Kim et al. [4] reported that multi wall carbon nanotubes induced DNA damage and cytotoxicity in male's human peripheral blood lymphocytes. Montes-Fonseca et al. [5] found cytotoxicity of functionalized carbon nanotubes in J774A macrophages. Sachar et al. [6] reported a cytotoxic effect of single wall carbon nanotubes (SWCNTs) on erythrocytes in vitro and in vivo. On the other hand, Professor Ballerini and her group made an elegant work in which they reported that MWCNTs were bio-compatible scaffolds for growing neuronal and myocardial mammalian cells [7] [8] [9] [10]. Fabbro et al. [7] [8] found that the adhesion to carbon nanotube conductive scaffolds forced the action-potential appearance in immature rat's spinal neurons; these findings allowed them to propose that carbon nanotubes could be used for neuroregeneration and repair. Recently, Bosi et al. [10] reported the elaboration of 3D nanostructured scaffolds to investigate signaling in reconstructed neuronal networks. Moreover, Martinelli et al. [9] reported that cardiac myocytes were cultured in carbon nanotubes' platforms and these nanotubes improved the cardiac myocytes' performance.

Pristine carbon nanotubes have hydrophobic properties and are difficult to disperse in biological aqueous solutions [11]. Polyvinylpyrrolidone (PVP) at different molecular weights, has been used as a dispersant for SWCNTs as well as MWCNTs, based on its non-toxicity and high compatibility with carbon nanomaterials [12] [13] [14] [15]. MWCNTs as well as SWCNTs have been labeled with fluorescent compounds in order to identify them in isolated cells and tissue [16] [17] [18] [19]. Shityakov et al. [16] reported that multi-walled carbon nanotubes functionalized with fluorescein isothiocyanate, were used to study the bloodbrain barrier's transport and molecular dynamics. Iancu et al. [17] used multiwalled carbon nanotubes functionalized with human serum albumin and labeled with fluorescein to enhance the laser's thermal ablation for the in vitro treatment of liver cancer. Hao et al. [18] reported the fluorescein delivery into plant cells using magnetic single-walled carbon nanotubes. Chen et al. [19] reported the incorporation of carbon nanotube-fluorescein conjugates in human ovarian cancer cells. However, Bosi et al. [10] reported that MWCNTs without fluorescent staining were visualized in white-gray color by confocal microscopy under reflection mode acquisition, allowing the visualization of 3D-PDMS-MWCNT scaffold structure. Due to the size and morphology of carbon nanotubes and their intrinsic hydrophobicity, it has been difficult to identify their direct interaction in aqueous biological media and with neuronal cell membrane.

In this work, we report an innovative experimental approach to identify MWCNTs deposited on the cerebral ganglia of the invertebrate Helix aspersa and over glass coverslip, without fluorescent staining. Based on the large size of spherical neurons $(100-150 \mu \mathrm{m})$ and the big size of MWCNTs (50 nm outside 
diameter and 10 - $50 \mu \mathrm{m}$ long), we were able to characterize the topographic and ultra-structural distribution of the MWCNTs deposited on the Helix aspersa neurons and over glass coverslip, by using Scanning Electron Microscopy (SEM) and Confocal Microscopy (CM) studies.

\section{Material and Methods}

\subsection{Synthesis, Functionalization and Dispersion of MWCNTs}

MWCNTs were prepared by spray pyrolysis technique developed by Aguilar-Elguezabal et al. [20]. MWCNTs were grown inside of quartz tubing furnace (Thermo Scientific) working at $800^{\circ} \mathrm{C}$, by spray pyrolysis of ferrocene/benzene under argon flow. Benzene acts as the carbon source (carbon precursor) and ferrocene as the catalyst. At the final stage of the synthesis, the thermocouple was turned off, leaving only the argon flow to prevent CNTs from rusting. Once the quartz tube was cooled down, CNTs were removed by scraping the inner tube with a spatula. MWCNTs were obtained with an average diameter of $70 \mathrm{~nm}$ and length of $70-$ $120 \mu \mathrm{m}$. They were functionalized by acidic treatment (nitric acid-sulfuric acid at a concentration of $4 \mathrm{M}$ and at 3:1 proportion). Polyvinylpyrrolidone (PVP-40) from Sigma-Aldrich was used in this work as a dispersant for MWCNTs in water solution. It was prepared according to the following formulation: $1 \mathrm{~g}$ PVP-40/100 $\mathrm{mg}$ of MWCNTs/20 $\mathrm{ml}$ of deionized water. With the aim to improve dispersion and to reduce the size of MWCNTs [21], MWCNTs-PVP stock solution ( $5 \mathrm{mg} / \mathrm{ml}$ ) was sonicated for 60 minutes by using a bath sonicator, Model VWR-50T. MWCNTs-PVP were re-suspended in snail ringer solution (SRS) prepared as follows: (in $\mathrm{mM}$ ) NaCl, 75; KCl, 5; $\mathrm{CaCl}_{2}, 10 ; \mathrm{MgCl}_{2}, 5$; Glucose, 5; HEPES, 5; $\mathrm{pH}$ 7.5. The final concentration of MWCNTs-PVP was $0.005,0.05$, and $0.5 \mathrm{mg} / \mathrm{mL}$.

\subsection{Biological Preparation}

The biological preparation used in this work was the cerebral ganglia of the garden snail Helix aspersa [22]. The Helix neurons have a spherical shape, size of 50 to 200 um and more than $90 \%$ of Helix neurons have spontaneous electrical activity; they do not need oxygenation, neither temperature control to keep them alive. The specimen was anesthetized with ethanol at $1 \%$ in SRS and the snail cerebral ganglia were obtained by microsurgery. The connective tissue that covers the ganglia was removed by micro-dissection. The ganglia were exposed to protease type XIV (Sigma-Aldrich) at $1 \mathrm{mg} / \mathrm{mL}$ in SRS for $15 \mathrm{~min}$ in order to facilitate the removal of the membrane attached to the nerve cells. The action of the enzyme was stopped by washing the ganglia three times with SRS. The fine membrane attached to the neurons was removed with microforceps to expose the nerve cells (see Figure 1(b)).

\subsection{Ultra-Structural Characterization of MWCNTs}

Scanning Electron Microscopy (SEM) and Confocal microscopy (CM) studies were done to study the morphology and topographic distribution of MWCNTs-PVP composite deposited over a glass coverslip and on the cerebral ganglia of Helix 


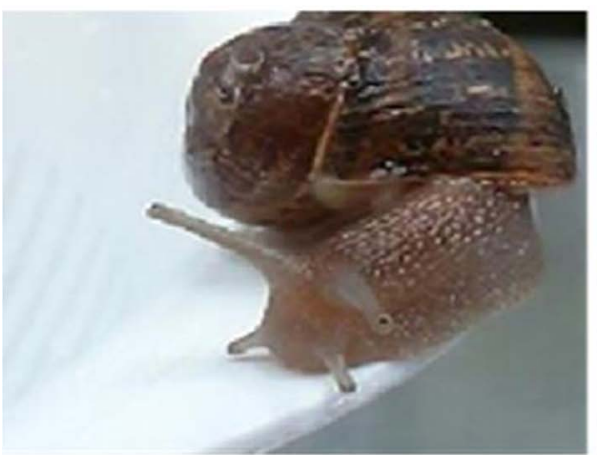

(a)

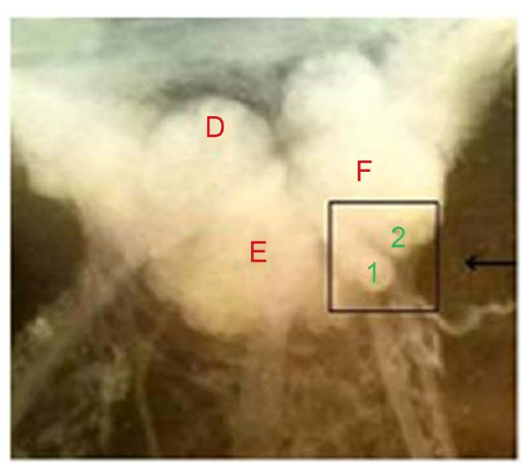

(b)

Figure 1. Biological preparation used in this work. (a) is the alive specimen and (b) is the isolated and dissected cerebral ganglia obtained from the specimen. D, E and F in red are the identified ganglia of Helix aspersa. $1 \mathrm{~F}$ and $2 \mathrm{~F}$ are the identified neurons in the inserted box. Scale bar represented by the arrow is $200 \mu \mathrm{m}$.

aspersa. For the SEM studies, we used a SEM microscope, Model JSM 5800, LV, with tungsten filament, at a maximum resolution of $3.5 \mathrm{~nm}$, with an accelerating voltage of $30 \mathrm{kV}$ and with a magnification from $45 \times$ to $300,000 \times$. All the samples studied with SEM (MWCNTs-PVP composite deposited over glass coverslip and on neurons) were previously treated with gold or silver by the sputtering method, using a Denton Vaccum Des II device. Samples of MWCNTs deposited on glass coverslip and over Helix neurons were studied and observed with a confocal microscope (Leica Microsystems GmbH, Wetzlar, Germany). Several images were acquired at $23 \times$ and $63 \times$ of magnification and serial confocal planes $(\mathrm{z}$ stack) were acquired every $10-100 \mathrm{~nm}$ across the entire 2D configuration. Reconstructions of the images were performed offline using the image-processing package Fiji [23]. MWCNTs were visualized by using the reflection mode property of the microscope, during the confocal acquisition. To prepare the MWCNTsPVP composite deposited on glass coverslip, we deposited $20 \mu \mathrm{L}$ of MWCNTsPVP at different dilutions (1, 1:10 and 1:100) on a rectangular glass coverslip (7 by $12 \mathrm{~mm}$ ). The composite was dried at room temperature for $12 \mathrm{~h}$ and then, it was heated for $12 \mathrm{~h}$ at $220^{\circ} \mathrm{C}$ in a convection oven.

\subsection{Helix aspersa Neurons Treated with MWCNTs-PVP}

MWCNTs were applied to the cerebral ganglia of Helix aspersa at final concentrations of $0.1 \mathrm{mg} / \mathrm{mL}$ in SRS and incubated for $12 \mathrm{hrs}$. To remove the non-attached nanotubes, the ganglia were washed two times with SRS. The ganglia were incubated and fixed in Paraphormaldehide at $4 \%$ in SRS for $12 \mathrm{~h}$ at room temperature. For SEM studies, after the ganglia were fixed, they were washed two times with SRS, and then they were deposited on brass porta samples and dried at room temperature for $12 \mathrm{~h}$. After that, samples were treated with silver by the sputtering method. In the confocal microscopy studies, cells were stained and treated as follows: first, cells were permeabilized with $1 \%$ Triton X-100 for $30 \mathrm{~min}$; second, for blocking the unspecific binding, the neurons were treated with $5 \%$ fetal bovine serum (FBS) in PBS blocking buffer for $30 \mathrm{~min}$ at room temperature; third, 
the ganglia were incubated for 30 min with DAPI (4',6-diamidino-2-phenylindole) for staining the nucleous and WGA-A594 (wheat germ agglutinin Alexa Fluor 594) for staining the cell membrane. DAPI is a green or blue-fluorescent DNA and it is excited by $405 \mathrm{~nm}$ laser line and is commonly used as a nuclear counter stain in fluorescence microscopy and confocal studies. The wheat germ agglutinin (WGA) is one of the most widely used in cellular biology lectins. The conjugated wheat germ agglutinin (WGA) Alexa Fluor 594 (WGA-A594) presents bright red dye (excitation/emission maximum of approx. 590/617 nm). Finally, ganglia samples were mounted on glass slide, treated with Vectashield (Vector Laboratories) and covered and sealed with coverslips of $1 \mathrm{~mm}$ thickness. Upon immunofluorescence staining, Helix aspersa neurons were studied and observed with confocal microscope.

\section{Results and Discussion}

Figure 2 shows the topographic distribution of MWCNTs-PVP at dilutions of 1:100 in SRS. It is observed that carbon nanotubes are well dispersed in this aqueous media and they are not agglomerated at all. This good dispersion of MWCNTs-PVP was corroborated when this composite was deposited on a glass coverslip and analyzed with confocal microscopy in the reflection mode. Independent nanotubes can be identified and are not aligned and distributed randomly (see Figure 3).

After the Helix aspersa neurons were treated with MWCNTs-PVP composite for $12 \mathrm{~h}$, in SEM studies, it was observed that the nanotubes were deposited on the surface of the neurons (Figure 4). Similar topographic distribution of MWCNTs-PVP composite was observed in neuronal cells when they were studied with the confocal microscope (Figure 5). In the micrograph presented in Figure 5, it was possible to observe that MWCNTs (in white-gray color) are attached to the neuronal membrane (stained in red), crossing the cell membrane and getting into the cytoplasm.

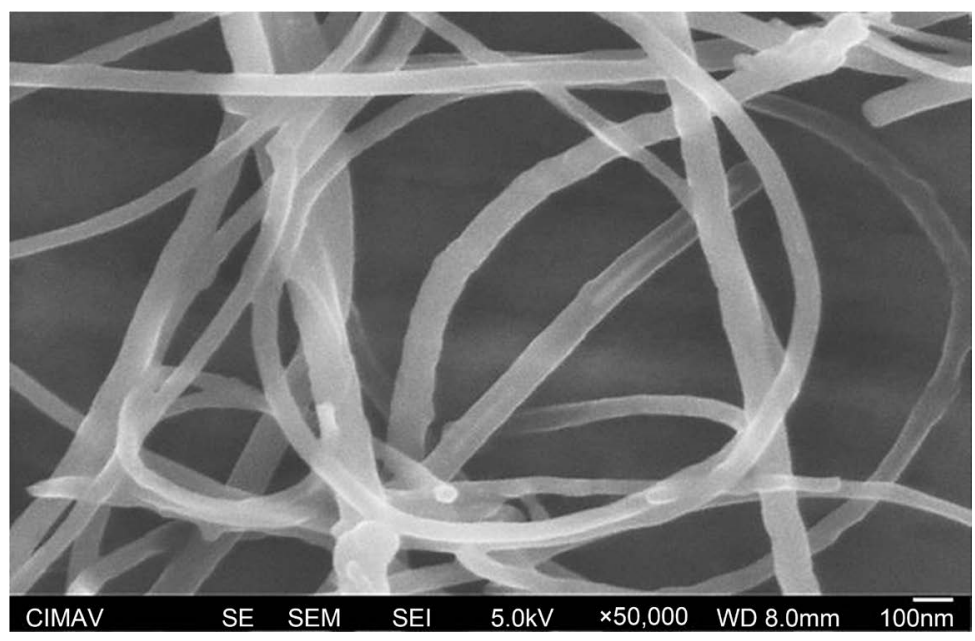

Figure 2. High resolution Scanning Electron Microscopy (SEM) micrograph to identify the topographic distribution of MWCNTs $(0.05 \mathrm{mg} / \mathrm{dl}$ in SRS) applied on a glass coverslip. Magnification $\times 50,000$, scale bar $100 \mathrm{~nm}$. 


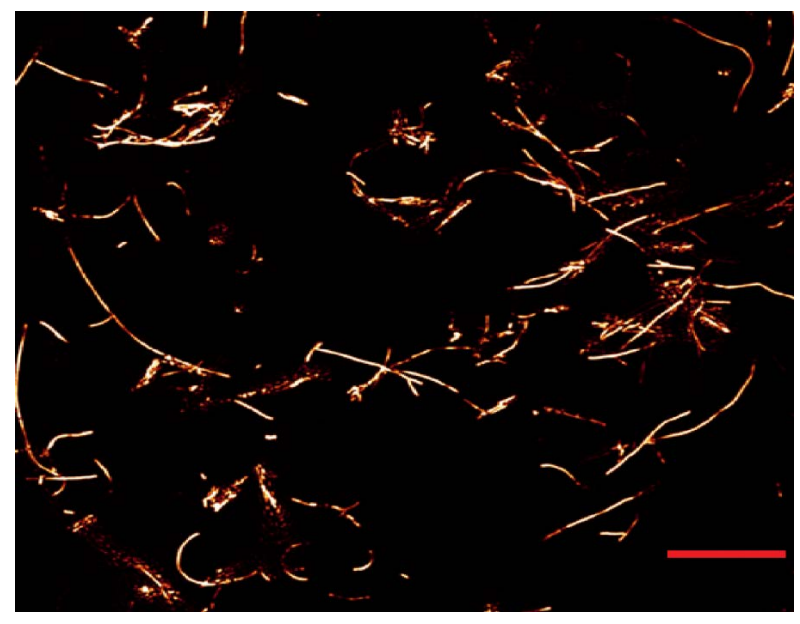

Figure 3. MWCNTs at $0.05 \mathrm{mg} / \mathrm{dl}$ in snail ringer solution deposited on glass coverslip and observed with the confocal microscope in the reflection mode at $460 \mathrm{~nm}$. Scale bar $10 \mu \mathrm{m}$.
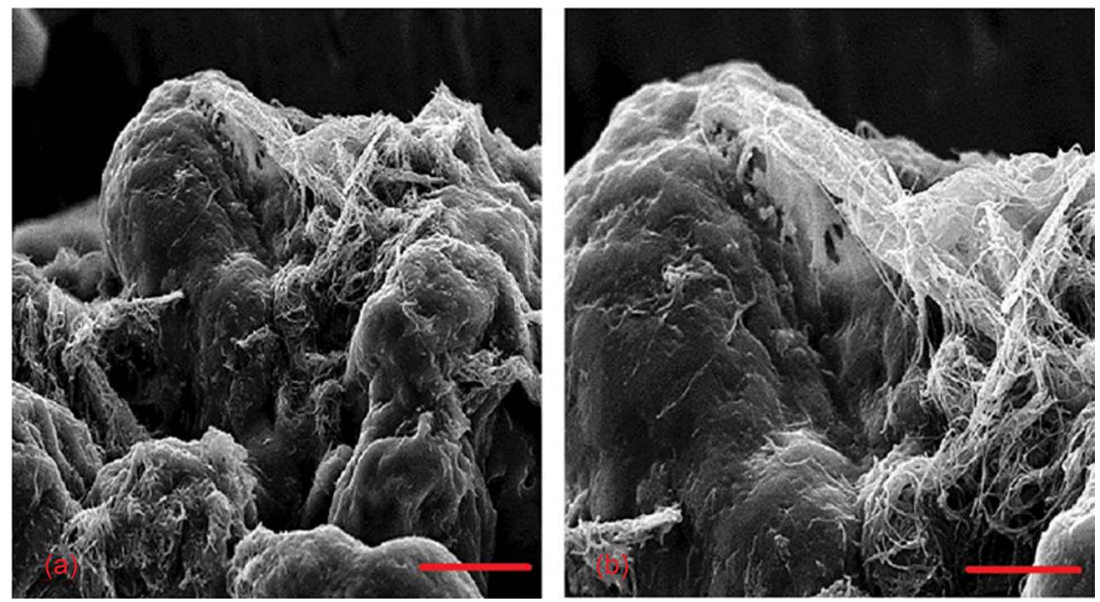

Figure 4. Low resolution Scanning Electron Microscopy micrograph to identify the topographic distribution of MWCNTs $(0.5 \mathrm{mg} / \mathrm{dl}$ in SRS) on the surface of Helix aspersa neurons. Scale bar $20 \mu \mathrm{m}$. (a) is at $1000 \times$ magnification and (b) is $3000 \times$ magnification.

Our results are in concordance with previous reports, in the sense that functionalized multi wall carbon nanotubes can be dispersible in aqueous solution by using specific dispersants [11] [12] [13] [14] [15]. Moreover, the results presented here corroborate the results reported by others [7] [8] [10], because in these experiments MWCNTs interact with living cells and act as functional scaf folds for neuronal tissue. An interesting finding reported here, is that it was no necessary to label the MWCNTs with fluorescent compounds, as it has been done and has been reported by others [5] [16] [17] [18] [19]. These MWCNTs were well identified with confocal microscopy studies (see Figure 3 and Figure 5) when the microscope was used in the reflection mode. This clear identification of MWCNTs deposited on glass coverslips and over snail neurons, can be explained by two facts. First, the MWCNTs used in this work were obtained with an average diameter of $50-70 \mathrm{~nm}$ and length of 50 to $120 \mu \mathrm{m}$ [20]. Second, the Helix aspersa ganglia have 


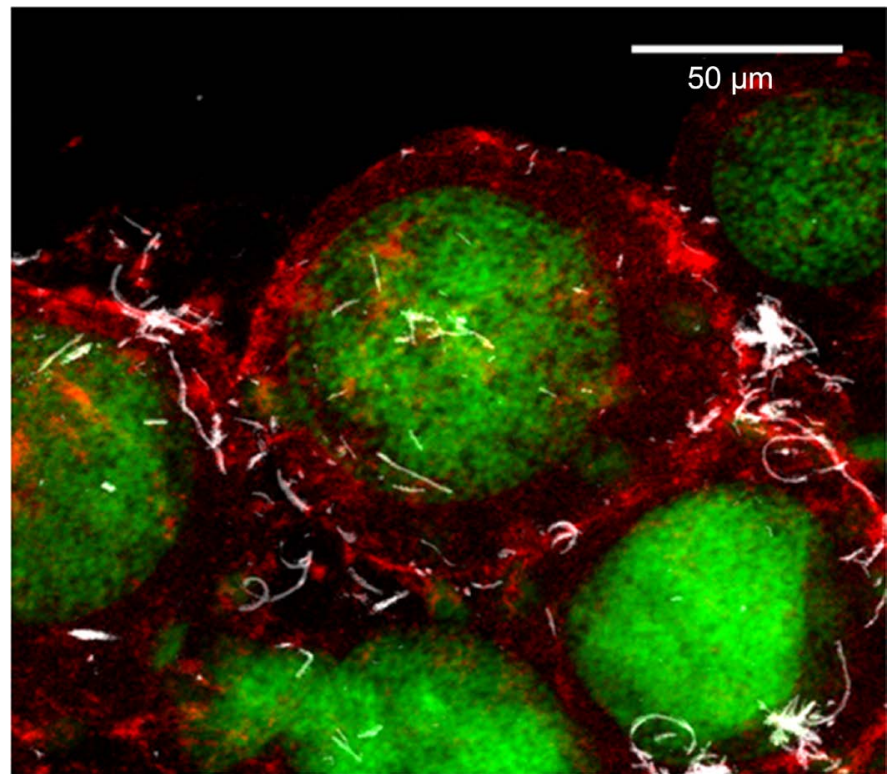

Figure 5. MWCNTs-PVP on Helix aspersa neurons. Neurons stained with WGA-A594 in red for cell membrane and DAPI in green, for nucleous. Gray-white color for MWCNTs in the reflection mode. 40× magnification, with zoom. Scale bar $50 \mu \mathrm{m}$.

spherical big neurons with a diameter between 50 - $200 \mu \mathrm{m}$ [22].

It has been reported that MWCNTs interact with the cellular membrane and have a blocking effect of membrane ion channels in cardiac cells [24] and neuronal cells as well [25] [26]. However, preliminary experiments reported by our group indicate that the MWCNTs, synthesized by spray pyrolysis, functionalized with acidic treatment and dispersed by PVP, have low electrical resistance and are good electro-conductive materials [14] [15]. Therefore, this MWCNT-PVP composite tested in this work may not block ion currents in neurons. Although in this work we found that carbon nanotubes have direct interaction with the neuronal cell membrane and can be useful for neuronal tissue engineering, we consider that more experimental work is needed in order to elucidate if these MWCNTs may modify specific ion channels immersed in the cellular membrane such as calcium, potassium and sodium channels, which are responsible for the electrical properties of neurons and other excitable cells.

\section{Conclusion}

In this work, we report that MWCNTs can be synthesized by spray pyrolysis, functionalized with acidic treatment and dispersed by PVP. We have demonstrated that the composite made of multi wall carbon nanotubes-polyvinylpyrrolidone (MWCNTs-PVP) has good dispersion properties in aqueous biological media, such as the snail ringer solution (SRS). Independent carbon nanotubes were clearly observed in SEM and CM studies, when they were applied on a glass coverslip. In addition, here we demonstrate that carbon nanotubes interact and get attached to the neuronal cell membrane. Therefore, this MWCNTs-PVP composite can act as a good scaffold for neurons in tissue culture. 


\section{Acknowledgements}

Authors are very grateful to Professor Laura Ballerini and her collaborators at International School for Advanced Studies (SISSA), Trieste, Italy, for the academic support in the performance of confocal microscopy studies. Juan BernalMartinez received a Scholar Fellowship by Conacyt-Mexico to support the present experimental work. Authors are also thankful to Ms. Teresa Villalobos, for the critical review of the manuscript.

\section{References}

[1] Harrison, B.S. and Atala, A. (2007) Carbon Nanotube Applications for Tissue Engineering. Biomaterials, 28, 344-353.

https://doi.org/10.1016/j.biomaterials.2006.07.044

[2] Abarrategia, A., Gutiérrez, M.C., Moreno-Vicente, C., Hortigüel, M.J., Ramos, V., López-Lacomba, J.L., Ferrer, M.L. and del Monte, F. (2008) Multiwall Carbon Nanotube Scaffolds for Tissue Engineering Purposes. Biomaterials, 29, 94-102. https://doi.org/10.1016/j.biomaterials.2007.09.021

[3] Haniu, H., et al. (2012) Basic Potential of Carbon Nanotubes in Tissue Engineering Applications. Journal of Nanomaterials, 2012, Article ID: 343747. https://doi.org/10.1155/2012/343747

[4] Kim, J.S., Song, K.S. and Yu, I.J. (2016) Multiwall Carbon Nanotube-Induced DNA Damage and Cytotoxicity in Male Human Peripheral Blood Lymphocytes. International Journal of Toxicology, 35, 27-37. https://doi.org/10.1177/1091581815598749

[5] Montes-Fonseca, S.L., Orrantia-Borunda, E., Aguilar-Elguezabal, A., González Horta, C., Talamás-Rohana, P. and Sánchez-Ramírez, B. (2012) Cytotoxicity of Functionalized Carbonnanotubes in J774A Macrophages. Nanomedicine, 8, 853-859.

[6] Sachar, S. and Saxena, R.K. (2011) Cytotoxic Effect of Poly-Dispersed Single Walled Carbon Nanotubes on Erythrocytes in Vitro and in Vivo. PLoS ONE, 6, e22032. https://doi.org/10.1371/journal.pone.0022032

[7] Fabbro, A., Prato, M. and Ballerini, L. (2013) Carbon Nanotubes in Neuroregeneration and Repair. Advanced Drug Delivery Reviews, 65, 2034-2044.

https://doi.org/10.1016/j.addr.2013.07.002

[8] Fabbro, A., Sucapane, A., Toma, F.M., Calura, E., Rizzetto, L., Carrieri, C., Roncaglia, P., Martinelli, V., Scaini, D., Masten, L., Turco, A., Gustincich, S., Prato, M. and Ballerini, L. (2013) Adhesion to Carbon Nanotube Conductive Scaffolds Forces Action-Potential Appearance in Immature Rat Spinal Neurons. PLOS ONE, 8, e73621. https://doi.org/10.1371/journal.pone.0073621

[9] Martinelli, V., Cellot, G., Fabbro, A., Bosi, S., Mestroni, L. and Ballerini, L. (2013) Improving Cardiac Myocytes Performance by Carbon Nanotubes Platforms. Frontiers in Physiology, 4, 239. https://doi.org/10.3389/fphys.2013.00239

[10] Bosi, S., Rauti, R., Laishram, J., Turco, A., Lonardoni, D., Prato, M., Scaini, D. and Ballerini, L. (2015) From 2D to 3D: Novel Nanostructured Scaffolds to Investigate Signalling in Reconstructed Neuronal Networks. Scientific Reports, 5, 9562. https://doi.org/10.1038/srep09562

[11] Hirsch, A. (2002) Functionalization of Single-Walled Carbon Nanotubes. Angewandte Chemie International Edition, 41, 1853-1859. https://doi.org/10.1002/1521-3773(20020603)41:11<1853::AID-ANIE1853>3.0.CO;2 -N

[12] Achaby, M.E., Arrakhiz, F.E., Vaudreuil, S., Essassi, M., Qaiss, M. and Bousmina, 
M. (2013) Nanocomposite Films of Poly(Vinylidene Fluoride) Filled with Polyvinylpyrrolidone-Coated Multiwalled Carbon Nanotubes: Enhancement of $b$-Polymorph Formation and Tensile Properties. Polymer Engineering and Science, 53, 34-43. https://doi.org/10.1002/pen.23236

[13] Nasouri, K., Shoushtari, A.M. and Mojtahedi, M.R.M. (2016) Synthesis and Characterization of Highly Dispersed Multi-Walled Carbon Nanotubes/Polyvinyl-Pyrrolidone Composite Nanofibers for EMI Shielding Application. Polymer Composites. https://doi.org/10.1002/pc.23774

[14] Bernal-Martínez, J., Seseña-Rubfiaro, A., Godínez-Fernández, R. and AguilarElguezabal, A. (2016) Electrodes Made of Multi-Wall Carbon Nanotubes on PVDFFilters Have Low Electrical Resistance and Are Able to Record Electrocardiograms in Humans. Microelectronic Engineering, 166, 10-14. https://doi.org/10.1016/j.mee.2016.09.006

[15] Bernal-Martínez, J., Godínez-Fernández, R., Roman-Aguirre, M. and Aguilar-Elguezabal, A. (2016) The Electrical Resistance of Electrodes Made of Multi Walled Carbon Nanotubes Is Modulated by nIR-Laser. Microelectronic Engineering, 166, 45-49. https://doi.org/10.1016/j.mee.2016.09.013

[16] Shityakov, S., Salvador, E., Pastorin, G. and Förster, C. (2015) Blood-Brain Barrier Transport, Aggregation, and Molecular Dynamics Simulation of Multiwalled Carbon Nanotube Functionalized with Fluorescein Isothiocyanate. International Journal of Nanomedicine, 10, 1703-1713. https://doi.org/10.2147/IJN.S68429

[17] Iancu, C., Mocan, L., Bele, C., Orza, A.I., Tabaran, F.A., Catoi, C., Stiufiuc, R., Stir, A., Matea, C., Iancu, D., Agoston-Coldea, L., Zaharie, F. and Mocan, T. (2011) Enhanced Laser Thermal Ablation for the in Vitro Treatment of Liver Cancer by Specific Delivery of Multiwalled Carbon Nanotubes Functionalized with Human Serum Albumin. International Journal of Nanomedicine, 6, 129-141. https://doi.org/10.2147/IJN.S15841

[18] Hao, Y., Yang, X., Shi, Y., Xing, J., Marowitch, J., Chen, J. and Chen, J. (2012) FITC Delivery into Plant Cells Using Magnetic Single-Walled Carbon Nanotubes. Journal of Nanoscience and Nanotechnology, 12, 6287-6293.

https://doi.org/10.1166/jnn.2012.6449

[19] Chen, M.T., Gomez, L.M., Ishikawa, F.N., Vernier, P.T., Zhou, C. and Gundersen, M.A. (2009) PH-Sensitive Intracellular Photoluminescence of Carbon NanotubeFluorescein Conjugates in Human Ovarian Cancer Cells. Nanotechnology, 20, Article ID: 295101. https://doi.org/10.1088/0957-4484/20/29/295101

[20] Aguilar-Elguezabal, A., Antunez, W., Alonso, G., Delgado, F.P., Espinosa, F. and Miki-Yoshida, M. (2006) Study of Carbon Nanotubes Synthesis by Spray Pyrolysis and Model of Growth. Diamond \& Related Materials, 15, 1329-1335.

https://doi.org/10.1016/j.diamond.2005.10.011

[21] Zaragoza-Contreras, E.A., Lozano-Rodríguez, E.D., Román-Aguirre, M., AntunezFlores, W., Hernández-Escobar, C.A., Flores-Gallardo, S.G. and Aguilar-Elgue-zabal, A. (2009) Evidence of Multi-Walled Carbon Nanotube Fragmentation Induced by Sonication during Nanotube Encapsulation via Bulk-Suspension Polymerization. Micron, 40, 621-627. https://doi.org/10.1016/j.micron.2009.02.007

[22] Kerkut, G.A., et al. (1973) Mapping of Nerve Cells in the Suboesophageal Ganglia of Helix Aspersa. Comparative Biochemistry and Physiology, 50, 1-25.

[23] Schindelin, J., et al. (2012) Fiji: An Open-Source Platform for Biological-Image Analysis. Nature Methods, 9, 676-682. https://doi.org/10.1038/nmeth.2019

[24] Tan, X.Q., Cheng, X.L., Zhang, L., Wu, B.W., Liu, Q.H., Meng, J., Xu, H.Y. and Cao, J.M. (2014) Multi-Walled Carbon Nanotubes Impair Kv4.2/4.3 Channel Activities, 
Delay Membrane Repolarization and Induce Bradyarrhythmias in the Rat. PLoS ONE, 9, e101545. https://doi.org/10.1371/journal.pone.0101545

[25] Chen, T., Yang, J., Ren, G., Yang, Z. and Zhang, T. (2013) Multi-Walled Carbon Nanotube Increases the Excitability of Hippocampal CA1 Neurons through Inhibition of Potassium Channels in Rat's Brain Slices. Toxicology Letters, 217, 121-128. https://doi.org/10.1016/j.toxlet.2012.12.013

[26] Xu, H., Bai, J., Meng, J., Hao, W., Xu, H. and Cao, J.M. (2009) Multi-Walled Carbon Nanotubes Suppress Potassium Channel Activities in PC12 Cells. Nanotechnology, 20, Article ID: 285102. https://doi.org/10.1088/0957-4484/20/28/285102

Submit or recommend next manuscript to SCIRP and we will provide best service for you:

Accepting pre-submission inquiries through Email, Facebook, LinkedIn, Twitter, etc. A wide selection of journals (inclusive of 9 subjects, more than 200 journals)

Providing 24-hour high-quality service

User-friendly online submission system

Fair and swift peer-review system

Efficient typesetting and proofreading procedure

Display of the result of downloads and visits, as well as the number of cited articles Maximum dissemination of your research work

Submit your manuscript at: http://papersubmission.scirp.org/

Or contact msce@scirp.org 